\title{
Formation of Lipoprotein-X
}

\section{ITS RELATIONSHIP TO BILE COMPOUNDS}

\author{
E. Manzato, R. Fellin, G. Baggio, S. Walch, W. Neubeck, and \\ D. SEIDEL
}

From the Department of Internal Medicine, Division of Gerontology and

Metabolic Disease, Policlinico, I-35100 Padua, Italy and Medizinische

Universitätsklinik (Ludolf Krehl Klinik), D-6900 Heidelberg, West Germany

\begin{abstract}
A в S T R A C T In this study we have demonstrated that in native bile, lipids are organized in the form of a lipoprotein (bile LP) carrying albumin as apoprotein. The lipid composition of bile LP is almost identical to lipoprotein-X (LP-X, the characteristic lipoprotein of cholestasis). However, it differs from LP-X in its protein/lipid ratio and immunological and electrophoretic characteristics. Bile lipoprotein can be converted into "LP-X-like" material in vitro by adding albumin or serum to native bile. The LP-X-like material formed in vitro has physicochemical and chemical characteristics similar or identical to LP-X isolated from serum. As bile lipoprotein can be converted into LP-X-like material by the addition of albumin to bile, LP-X can be converted into bile-LP-like particles by adding bile salts to a LP-X-positive serum. Furthermore, experimental connection of the common bile duct to the vena cava is followed after a few hours by the appearance of LP-Xlike material in the plasma. These facts taken together strongly suggest that bile LP is a precursor lipoprotein for LP-X and that it refluxes into the plasma pool under cholestatic conditions.
\end{abstract}

\section{INTRODUCTION}

It has been well documented that the characteristic elevation of unesterified cholesterol and phospholipids in patients with cholestasis is due to the presence of a low-density lipoprotein $(d 1.019-1.063 \mathrm{~g} / \mathrm{ml})$ of abnormal composition and properties. This lipoprotein is

Please address correspondence and requests for reprints to Dr. Dietrich Seidel, Medizinische Universitätsklinik (Ludolf Krehl Klinik), Bergheimerstrasse 58, D-69 Heidelberg, West Germany.

Received for publication 19 May 1975 and in revised form 29 December 1975. designated "lipoprotein X" (LP-X) ${ }^{1}(1-9)$. One important and characteristic feature of LP-X is its mobility towards the cathode on agar gel electrophoresis. This provided the basis for a simple analytical technique (10) to demonstrate LP-X in plasma samples; its appearance has been used as a new diagnostic test in the differential diagnosis of cholestatic liver disease. Several studies in adults and children (11-22) have demonstrated that the presence of this lipoprotein is an extremely specific sign of cholestasis, with the sole exception of the rare familial disease lecithin:cholesterol acyltransferase deficiency (23-25).

Although many data have accumulated in the past regarding the protein-lipid composition of LP-X, its immunological and physicochemical characteristics, its electrophoretic behavior in various media, and its structural properties (1-9), little or no information is available regarding the source of LP-X, its metabolism, or its fluctuations in the course of cholestatic liver disease. We therefore conducted a series of studies with a view to investigating these questions. In this report we provide evidence that LP-X most likely derives from a "precursor" lipoprotein complex which is normally excreted by the liver into bile, but which refluxes under cholestatic conditions into the plasma pool where it appears as LP-X.

\section{METHODS}

Collection of bile. Bile samples were obtained from patients with indwelling T-tubes 1-2 wk after cholecystectomy for gallstone disease (23 patients) or during operation from

${ }^{1}$ Abbreviations used in this paper: apo A, B, and C, apolipoproteins $\mathrm{A}, \mathrm{B}$, and $\mathrm{C}$, respectively; bile $\mathrm{LP}$, bile lipoprotein; HDL, high-density lipoprotein; LDL, low-density lipoprotein ; LP-X, lipoprotein-X; VLDL, very low-density lipoprotein. 
3 patients with cancer of the pancreas. All patients suffering from gallstone disease had normal plasma bilirubin levels and were LP-X negative. After collection, the bile was always kept at $4^{\circ} \mathrm{C}$ before further use.

Animal experiments. Four male beagle dogs (weight range $8.5-11.0 \mathrm{~kg}, 1-2 \mathrm{yr}$ ) were used for the animal experiments. Anesthesia was performed with Nembutal, $20 \mathrm{mg} / \mathrm{kg}$ (Deutsche Abbott GmbH., Ingelheim, W. Germany). In an operation lasting $30 \mathrm{~min}$ the bile duct was inserted into the inferior vena cava with an end-to-side anastomosis. Blood samples were collected from a peripheral vein before operation, shortly before setting the anastomosis, $30 \mathrm{~min}$ after the operation, and then every subsequent hour over a period of $26 \mathrm{~h}$.

Chemical analyses. LP-X was qualitatively determined with the "Rapidophor all in for LP-X" (a commercially available test kit by Immuno AG, Vienna, Austria) or with the use of $1 \%$ Bacto agar (Difco Laboratories, Detroit, Mich.) gel plates which we prepared ourselves as previously described $(3,10)$. The quantitation was performed according to our recently described procedure (26), which is based on densitometric scanning of the characteristic LP-X band after agar electrophoresis in the Rapidophor system. Agarose and agar electrophoreses were performed as described elsewhere (27); the lipoprotein bands were visualized by the use of polyanionic compounds $\left(0.2 \mathrm{M} \mathrm{CaCl}_{2}: 0.6 \%\right.$ sodium-dextran sulphate 500 or $0.1 \mathrm{M} \mathrm{MgCl}_{2}: 0.15 \%$ sodium heparin: $1.3 \% \mathrm{NaCl}$ ) (28). Immunological properties of the various fractions were studied by double diffusion and immunoelectrophoresis in $1 \%$ agar (Bacto agar, Difco Laboratories) or $0.8 \%$ agarose (Serva, Heidelberg, W. Germany) gels employing a barbital buffer, $\mathrm{pH} 8.6$, ionic strength 0.05 . The plates were developed as previously described (29). Rabbit antisera to apolipoprotein A (apo A; consisting of the $A_{1}$ and $A_{2}$ peptides), apolipoprotein $B$ (apo $B$ ) and apolipoprotein $C$ (apo $C$; consisting of the $C_{1}, C_{2}$, and $C_{3}$ peptides) were prepared in our own laboratory (29) and carefully tested for their specificity by employing immunoelectrophoretic techniques on human plasma, isolated homogeneous lipoprotein fractions, and apolipoprotein preparations characterized by polyacrylamide gel electrophoresis (29). Antibodies to human albumin and human gamma globulins were purchased from Behringwerke AG (Marburg/Lahn, W. Germany). Quantitation of albumin was also performed according to the Mancini technique employing commercially available test plates (Behringwerke AG). Esterified and unesterified cholesterol, phospholipids, and proteins were determined as described earlier $(3,4)$. Triglycerides were measured enzymatically by using a standard procedure (Boehringer GmbH., Mannheim, W. Germany). Bile acids were measured enzymatically with the Sterognost$3 \alpha$ (Nyegaard \& Co. AS, Oslo, Norway).

Isolation of a bile-lipoprotein complex. In order to isolate the bile-lipoprotein complex, native or water-dialyzed $(24 \mathrm{~h})$ bile was first centrifuged for $20 \mathrm{~min}$ at $20,000 \mathrm{rpm}$, $4^{\circ} \mathrm{C}$. The supernatant fraction was separated from a pellet (which was not further characterized) and submitted to polyanion precipitation. To $100 \mathrm{ml}$ of bile $\mathrm{MnCl}_{2}$ (final concentration $0.1 \mathrm{M}$ ) and sodium-dextran sulphate 500 (final concentration $0.5 \%$ ) was added and the solution adjusted to $\mathrm{pH} 7.0$ by the dropwise addition of $1 \mathrm{~N} \mathrm{NaOH}$. The mixture was then allowed to precipitate for $3 \mathrm{~h}$ at room temperature and submitted to centrifugation for $30 \mathrm{~min}$ at $20,000 \mathrm{rpm}$. The precipitate was resuspended in $50 \mathrm{ml}$ Trisbuffer ( $\mathrm{pH} 7.7,0.005 \mathrm{M}$ ) containing $0.1 \%$ dextran sulphate 500 and $0.1 \mathrm{M} \mathrm{MnCl}_{2}$ under constant stirring for $30 \mathrm{~min}$ and again centrifuged for $10 \mathrm{~min}$ at $6,000 \mathrm{rpm}$. This washing procedure was repeated twice. The final precipitate was dissolved in $20 \mathrm{ml}$ of a solution containing $2 \%$ sodium citrate and $1 \% \mathrm{NaCl}$; the $\mathrm{pH}$ of this solution was adjusted to 8 with $1 \mathrm{~N} \mathrm{NaOH}$. After gentle stirring for $30 \mathrm{~min}$ the solution was again centrifuged for $10 \mathrm{~min}$ at $6,000 \mathrm{rpm}$ and the supernatant fraction containing the dissolved bile lipoprotein was finally dialyzed against Tris buffer $(0.005 \mathrm{M})$ containing $0.9 \% \mathrm{NaCl}$ and stored at $4^{\circ} \mathrm{C}$ before use. To establish the hydrated density of the bile lipoprotein, native bile was also submitted to sequential ultracentrifugation with a type 60 rotor and the Heraeus Christ (Osterode, W. Germany) ultracentrifuge, type Omega II. Each centrifugation was performed at $150,000 \mathrm{~g}$ at $10^{\circ} \mathrm{C}$ for $24 \mathrm{~h}$. The first centrifugation was performed at a solution density of $1.006 \mathrm{~g} / \mathrm{ml}$; the bottom fraction containing the bile lipoprotein was recentrifuged twice at a density of $1.063 \mathrm{~g} / \mathrm{ml}$ yielding the bile lipoprotein (designated "bile LP") in the top fraction

In vitro formation of $L P-X$ and its isolation. Various forms of LP-X-like material were obtained when native bile or water-dialyzed $(24 \mathrm{~h})$ bile was incubated with various amounts of human albumin devoid of lipids ("Human albumin dried, purified," Behringwerke AG) or with total human serum. For the in vitro formation of LP-X-like material only a very short incubation time (less than $1 \mathrm{~min}$ ) at room temperature is required. The bile/albumin or bile/ serum ratio may vary from bile sample to bile sample depending on both bile salt and bile lipoprotein concentration (see under Results and Fig. 4). For special experiments ${ }^{125}$ I-albumin obtained from Amersham Buchler $\mathrm{GmbH}$ (Wenden, W. Germany) was used for LP-X formation. After incubation the mixtures were analyzed for the presence of LP-X (Rapidophor-agar electrophoresis) and submitted to the procedure of LP-X isolation.

Since the addition of albumin or serum to the isolated bile LP did not result in LP-X formation, bile salts (equal amounts of the taurine conjugates of cholic acid and chenodeoxycholic acid) in a final concentration of $100 \mathrm{mg} / 100 \mathrm{ml}$ were added to the bile LP before incubation with the protein solution. This then resulted in formation of LP-X-like particles.

LP-X from total serum was isolated in principle as described earlier (3). The serum was first submitted to ultracentrifugation at a solution density of $1.035 \mathrm{~g} / \mathrm{ml}$ and the bottom fraction containing LP-X was separated by the tube slicing techniques and further fractionated by cold ethanol precipitation (Cohn fractionation) as previously described (3). The supernatant fraction, corresponding to Cohn fraction IV-VI, was again submitted to ultracentrifugation at a solution density of $1.063 \mathrm{~g} / \mathrm{ml}$. The top fraction contained only intact LP-X in concentrated form.

LP-X-like particles formed after incubation of native or water-dialyzed bile with human albumin, designated "LP$\mathrm{X}_{(\mathrm{a} \mid \mathrm{b})}, "$ were isolated by sequential ultracentrifugation. The bile-albumin mixture was first submitted to ultracentrifugation (conditions identical to those described for isolation of the bile lipoprotein) at a solution density of 1.03 $\mathrm{g} / \mathrm{ml}$. The bottom fraction, which contained the LP-X (alb), was recentrifuged at a solution density of $1.09 \mathrm{~g} / \mathrm{ml}$ and the top fraction was recentrifuged again at a solution density of $1.09 \mathrm{~g} / \mathrm{ml}$ in order to remove the last traces of free albu$\min$. LP-X $(a \mid b)$ was then present in the top fraction. LP $\mathrm{X}_{\text {(alb) }}$ (final concentration $300 \mathrm{mg} / 100 \mathrm{ml}$ ) was also incubated with isolated very low-density lipoprotein (VLDL) and high-density lipoprotein (HDL) (final concentration $\sim 250$ and $\sim 150 \mathrm{mg} / 100 \mathrm{ml}$, respectively) for $2 \mathrm{~h}$ at room temperature and afterwards separated by ultracentrifugation 
NATIVE BILE
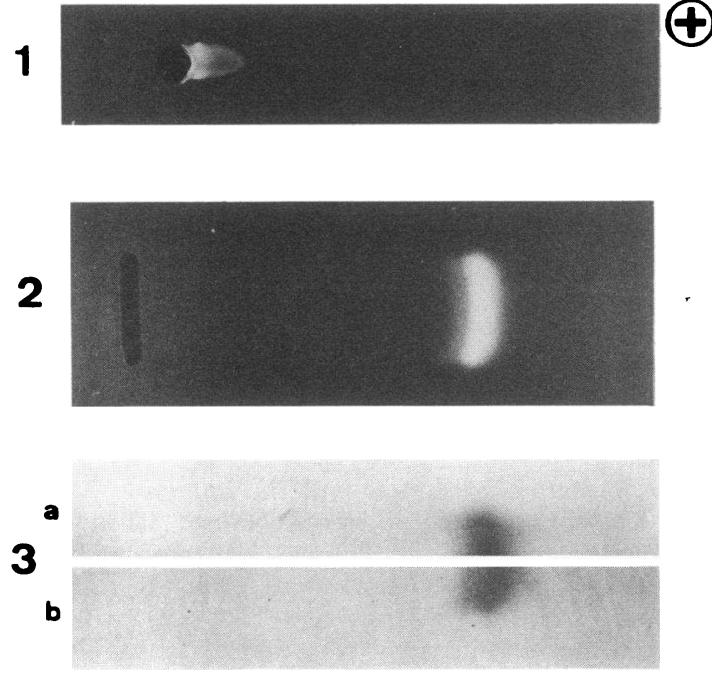

4

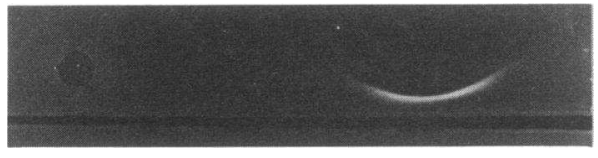

5
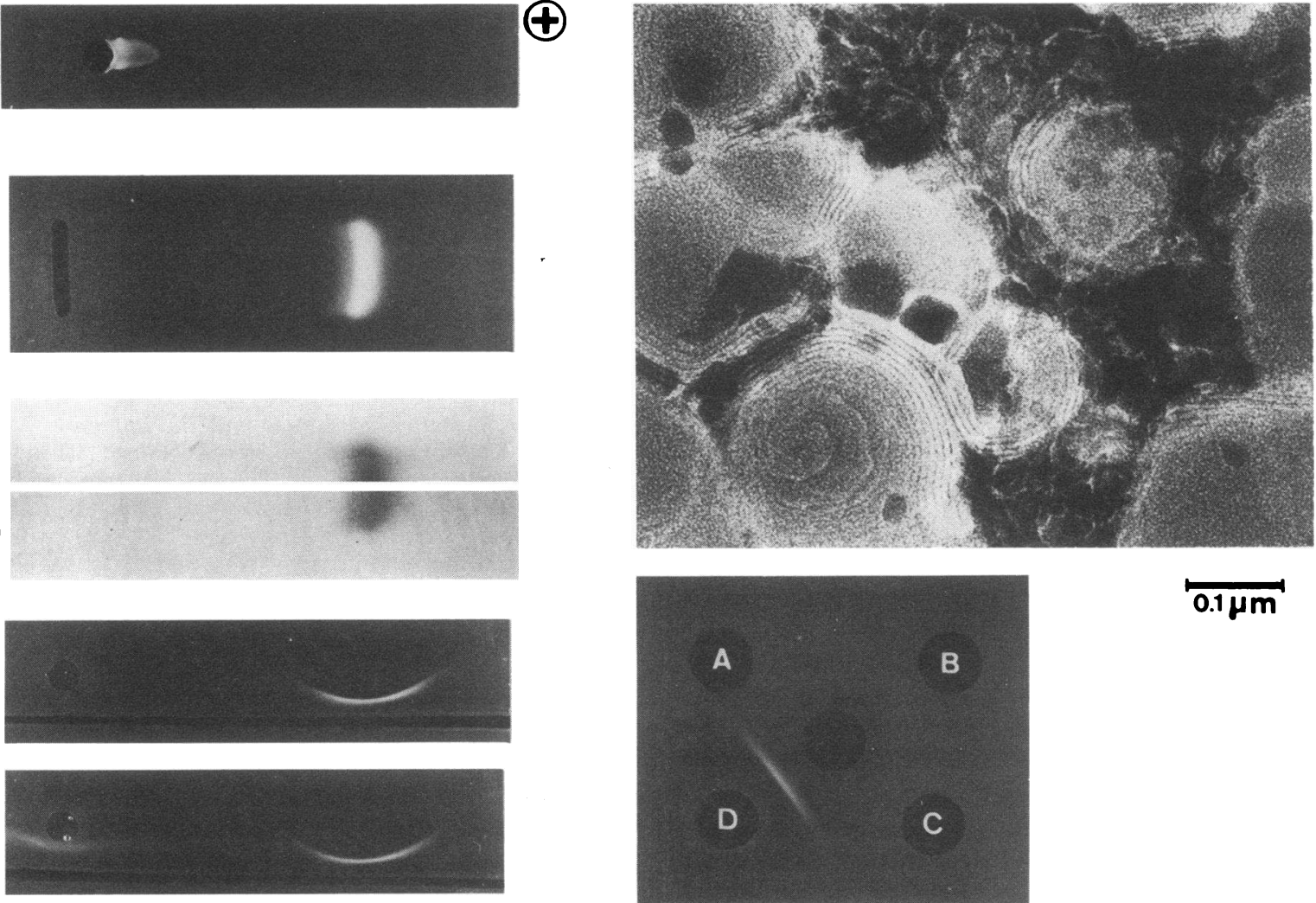

$0.1 \mu \mathrm{m}$

\section{ISOLATED LP-X}

6

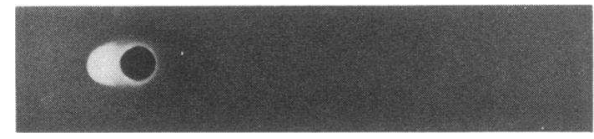

7

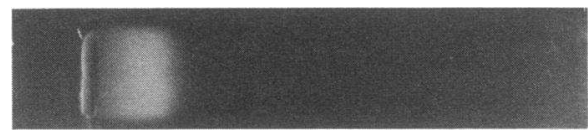

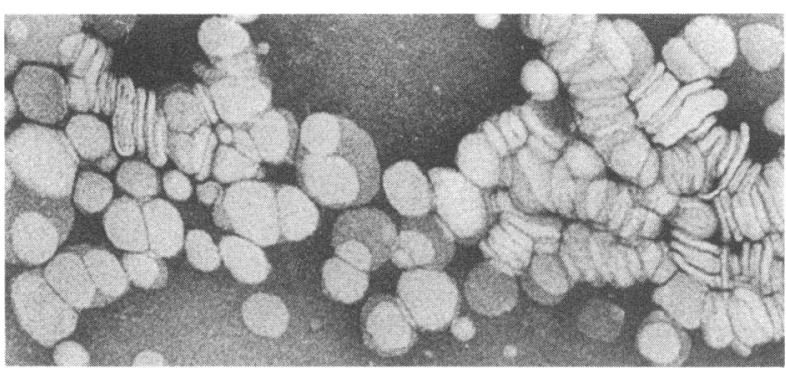

$0.1 \mu \mathrm{m}$

FIGURE 1 Lipoprotein pattern, immunological characteristics, and electron microscopy of a native human bile sample (1-5) in comparison with LP-X isolated from a patient with cholestatic liver disease (6 and 7) (protein-lipid composition (milligrams/100 ml) of the bile sample demonstrated in the figure: phospholipid, 179; cholesterol, 84; triglyceride, 10; protein, 120). Lipoprotein electrophoresis in $1 \%$ agar (1) and $0.8 \%$ agarose gel (2 and 3 ) clearly reveals the presence of a lipoprotein in native bile, which can be visualized with polyanionic compounds ( $0.1 \mathrm{M} \mathrm{MgCl}_{2}: 0.15 \%$ sodium heparin: $1.3 \% \mathrm{NaCl}$ ) (1 and 2) or with sudan black $(3 a)$ and with amido black $(3 b)$. Slide no. 3 was cut in two parts after electrophoresis in order to show that each half of the strip was stained separately for protein and lipid. The 
at the densities of $1.006 \mathrm{~g} / \mathrm{ml}$ or $1.063 \mathrm{~g} / \mathrm{ml}$, respectively. LP-X formed after incubation of native or water-dialyzed bile with total serum, designated "LP-X(ser)," was isolated by the procedure identical to that described for LP-X isolation from serum by using the combination of sequential ultracentrifugation and cold ethanol precipitation.

Isolation of $V L D L$, low-density lipoprotein $(L D L)$, and $H D L$. Normal plasma lipoprotein fractions were isolated and purified by sequential ultracentrifugation at the hydrated densities of $1.006,1.063$, and $1.21 \mathrm{~g} / \mathrm{ml}$. All fractions were washed twice by recentrifugation. The ultracentrifugation was performed under conditions identical to those described for the isolation of bile LP.

Partial delipidation. Partial delipidation of the isolated lipoprotein fractions was performed as described earlier with cold $n$-heptane on lyophilized material (4).

Determination of bile concentration. To compare native bile from various donors, bile concentration (dry weight) was determined gravimetrically before and after dialysis against water after lyophilization of portions of the sample.

Electron microscopy. The electronmicroscopy was performed on various lipoprotein fractions and native bile after negative staining at $25^{\circ} \mathrm{C}$ with a $1 \%$ aqueous solution of phosphotungstic acid adjusted to $\mathrm{pH} 7.1$ with $\mathrm{KOH}$ according to a standard procedure previously described in detail (8). The grids were examined in a Zeiss EM 10 electron microscope (Carl Zeiss, Oberkochen, W. Germany).

\section{RESULTS}

Human bile (Fig. 1) submitted to agar or agarose electrophoresis after dialysis against water reveals a lipoprotein band migrating towards the anode, which can be visualized by polyanion precipitation $\left(\mathrm{MgCl}_{2}+\right.$ sodium heparin or $\mathrm{CaCl}_{2}+$ dextran sulphate) as well as with a lipid stain (Sudan black) and protein stain (amido black). Before dialysis such bands may show some trailing or even be divided into several bands. Immunochemically, albumin and immunoglobulins as well as other plasma proteins (30) can be identified, but no immunoprecipitation reaction is obtained with antibodies to the major apolipoproteins: apo A, apo B, and apo $C$. Under the electron microscope it is not possible to detect reproducibly any particular structures. Occasionally some lamellar structures are apparent which may form myelinic shapes reminiscent of those earlier described for micellar solutions of lipids (31). This is in accordance with previous studies performed by Nalbone et al. (32). LP-X, with its characteristic structure under the electron microscope $(7,8)$ or, even more specifically, with its typical migration toward the cath- ode on agar electrophoresis (3), was detected in neither native nor water-dialyzed bile.

All $(100 \%)$ of bile lipids may be separated from bile by polyanion precipitation or by ultracentrifugation in form of an albumin-lipid complex (the bile LP) (Fig. 2). After separation of the bile LP the remaining bile fraction no longer contains lipids in measurable amounts nor does it show a lipoprotein band on electrophoresis. However, free albumin remains in this fraction.

The isolated and purified bile LP revealed a lipid composition quite similar to that of total bile and to that of LP-X isolated from patients suffering from cholestatic liver disease (Table I). The total bile salt concentration of isolated bile LP was found to range between 1 and $3 \%$ on a weight basis. The hydrated density of bile LP, which was found to be in the range $1.006-1.063 \mathrm{~g} / \mathrm{ml}$, is also similar to LP-X. In contrast to LP-X, however, isolated bile LP does not migrate on agar or agarose electrophoresis. In its intact and pure form it does not react immunochemically with antialbumin serum. However, the addition of bile salts to the isolated bile LP results in marked changes in its properties. The bile LP in a solution of bile salts (final concentration $250 \mathrm{mg} / 100 \mathrm{ml}$ ) not only migrates on electrophoresis in the same position as it does in native bile but it also reacts immunochemically with antialbumin serum. This immunoreaction may also be obtained with the isolated nonreactive bile LP after partial delipidation with $n$-heptane, indicating that the immunoreactive portion of albumin is covered by lipids in the isolated bile LP. This behavior has been described earlier for LP-X isolated from serum (4).

Agar electrophoresis (Fig. 3) demonstrates clearly that native and water-dialyzed bile, isolated albumin, and whole serum obtained from healthy volunteers do not show the typical lipoprotein band for LP-X migrating towards the cathode on agar electrophoresis. However, when native or water-dialyzed bile is incubated in vitro with either pure albumin or with total (LP-X negative) serum, LP-X is formed with an electrophoretic behavior identical to that found in the serum of patients suffering from cholestatic liver disease.

Addition of various amounts of isolated VLDL, LDL, or HDL (final concentrations ranging from 300 to 1,200 $\mathrm{mg} / 100 \mathrm{ml}$ for each fraction) as well as purified im-

electrophoretic mobility of the bile LP is in the albumin position as judged by electrophoresis in $0.8 \%$ agarose (4 and 5) against antialbumin serum (4) and anti-human serum (5). On double immunodiffusion native bile (central well) shows no precipitin line with antibodies to apo A (A), apo B (B) or apo C (C) but reacts with antialbumin (D) and other plasma proteins (5). On the electron micrograph some lamellar structures which may assume a myelinic shape are apparent. LP-X with its characteristic structure under the electron microscope, or with its typical migration towards the cathode on agar electrophoresis (6) and towards the anode on agarose electrophoresis (7) cannot be detected in bile. 


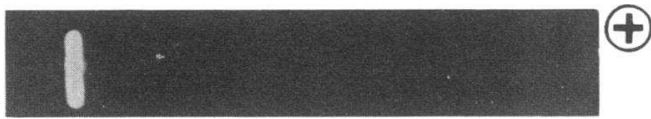

2

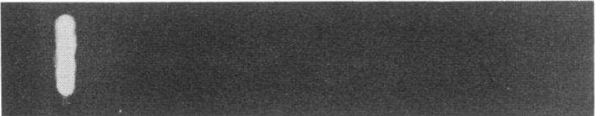

3

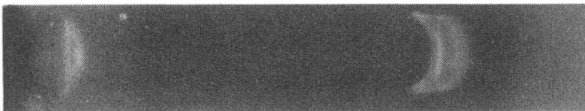

4

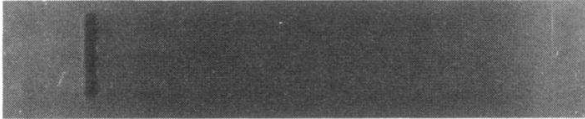

5
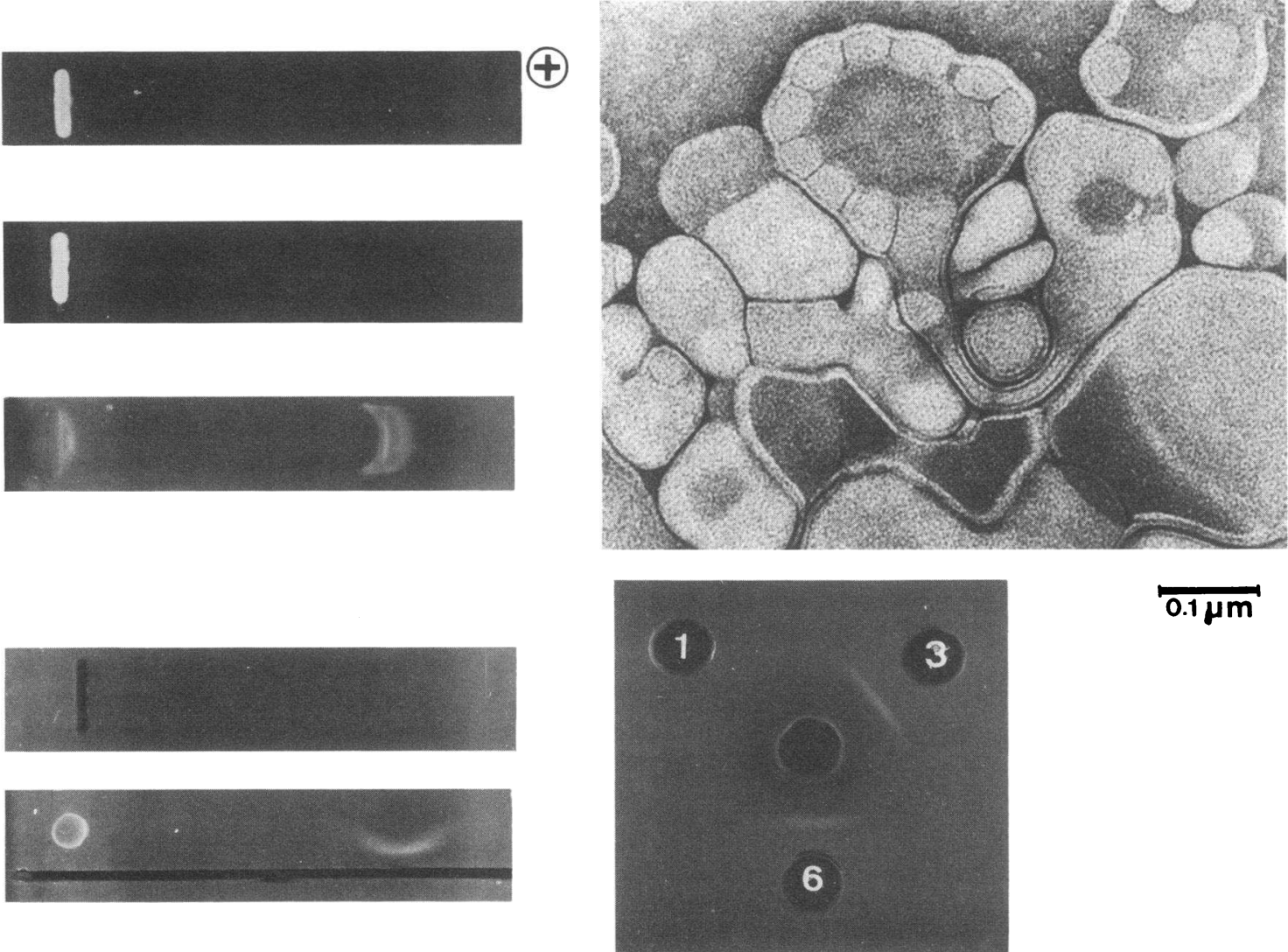

$0.1 \mu \mathrm{m}$

FIGURE 2 Characteristics of isolated bile LP (precipitation technique) and of the remaining bile fraction. The isolated, intart bile LP does not develop electrophoretic mobility on either $1 \%$ agar gel (1) or $0.8 \%$ agarose gel (2) electrophoresis. On patterns $1-4$ the lipoprotein bands were visualized by polyanionic compounds $\left(0.1 \mathrm{M} \mathrm{MgCl}_{2}: 0.15 \%\right.$ sodium heparin: $1.3 \%$ $\mathrm{NaCl}$ ). The isolated fraction remains at the origin (1 and 2) and shows no immunochemical reaction with antialbumin serum (immunodiffusion pattern 1 ; central well contains antialbumin serum). The bile fraction remaining after removal of the bile LP does not show any lipids or lipoprotein bands (4) but reacts with antialbumin serum (5). Addition of bile salts $(250 \mathrm{mg} /$ $100 \mathrm{ml}$ ) to the isolated bile LP induces the original electrophoretic mobility on agarose gel (3) and leads to an immunoreaction with antialbumin serum (immunodiffusion pattern 3 ). This immunoreaction may also be obtained after partial delipidation of the isolated bile LP (immunodiffusion pattern 6). On the electron micrograph isolated bile LP in suspension of bile salts shows particles irregular in size and shape; some lamellar structures may be visualized.

TABLE I

Lipid Composition and Protein/Lipid Ratio of Native Human Bile, Isolated Bile LP, and Various Forms of $L P-X$

\begin{tabular}{lccccc}
\hline & Phospholipid & $\begin{array}{c}\text { Free } \\
\text { cholesterol }\end{array}$ & $\begin{array}{c}\text { Cholesterol } \\
\text { ester }\end{array}$ & Triglyceride & Protein/lipid \\
\hline & $\%$ & $\%$ & $\%$ & $\%$ & $w t / w t$ \\
Bile & 63 & 27 & 3 & 7 & 0.42 \\
LP-X & 70 & 24 & 3 & 3 & 0.06 \\
Bile LP & 71 & 20 & 3 & 6 & 0.02 \\
LP-X (alb) & 74 & 21 & 2 & 3 & 0.15 \\
LP-X $_{(\text {alb }+ \text { VLDL) }}$ & 74 & 19 & 2 & 5 & 0.10 \\
LP-X $_{\text {(ser) }}$ & 66 & 26 & 2 & 6 & 0.03 \\
\hline
\end{tabular}




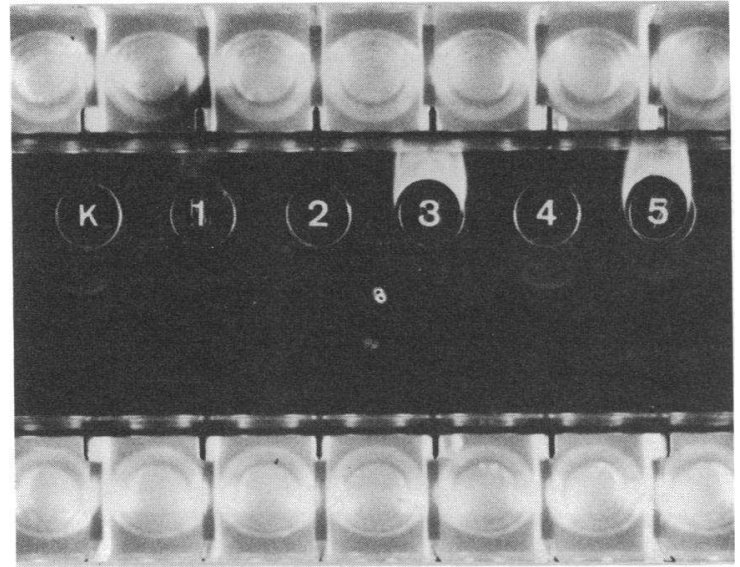

FIgURE 3 Agar electrophoresis (Rapidophor system) of LP-X-positive control sample of the test kit $(K)$, native bile (1), human albumin (2), LP-X-negative serum (3), mixture of bile and albumin (4), and mixture of serum and bile (5). While native bile, albumin, and LP-X-negative serum show no lipoprotein band typical for LP-X, the mixtures of bile and albumin or bile and serum develop the characteristic LP-X band.

munoglobulins to native bile does not result in LP-X formation.

The amount of LP-X formation from bile after addition of albumin or serum depends to a high degree on the concentration of the native bile and in particular on the concentration of bile LP and bile salts. This becomes apparent when the ratio of bile/ albumin necessary for LP-X formation is evaluated. The ratios for different bile samples from various donors before and after water dialysis are reported in Fig. 4. The lines given in Fig. 4 indicate the minimum amount of albumin required for LP-X formation at a certain bile concentration. In each instance the amount of LP-X formation will increase with increasing concentrations of albumin added to the bile. Marked differences are found before dialysis, while after dialysis all bile samples form a single line. During 36-h dialysis against water we found that up to $97 \%$ of the bile acids were dialyzable from native bile, while all lipids remained in the dialysis tube.

LP-X formed after addition of albumin to bile (LP$\mathrm{X}(\mathrm{a} \mid \mathrm{b})$ ) and after addition of serum (LP-X(ser)) may be isolated as described in Methods. Comparison of LP$\mathrm{X}$ (alb) and LP-X(ser) with isolated bile LP and LP-X isolated from serum revealed a very similar lipid composition (Table I). Significant differences are apparent, however, in their protein moieties and in their structural properties as judged by electron microscopy and electrophoresis on agar gel.

As demonstrated in Fig. 5 addition of albumin to bile in appropriate amounts leads to a complete conversion of the bile LP into LP-X(alb). While native bile shows a strong lipoprotein band migrating towards the anode on agar electrophoresis, which was earlier identified as bile LP, the mixture of bile plus albumin reveals only the typical LP-X band. In contrast to normal LP-X, LP-X (alb) does not react immunochemically with either anti-apo $C$ nor with antialbumin serum. After partially delipidation of LP-X(alb), however, an immunoreaction with antialbumin serum develops. This is also true for the isolated bile LP and LP-X isolated from serum.

In order to evaluate whether the albumin added to bile only binds free bile salts or is also utilized for the formation of LP-X, ${ }^{125}$ I-labeled albumin was added to native bile in separate experiments and the resulting LP-X (alb) separated from the remaining fraction. The specific activities found in LP-X(alb) after separation and in the remaining bile fractions were almost identical $(80,000)$ when expressed in terms of counts/milligram albumin of LP-X(alb) per min and counts/milligram albumin per min of the remaining bile fraction. This indicates that the albumin added is utilized for LP-X formation.

Although the amount of albumin bound to bile LP when this is converted to LP-X-like particles seems to be rather constant, as judged from the protein/lipid ratio

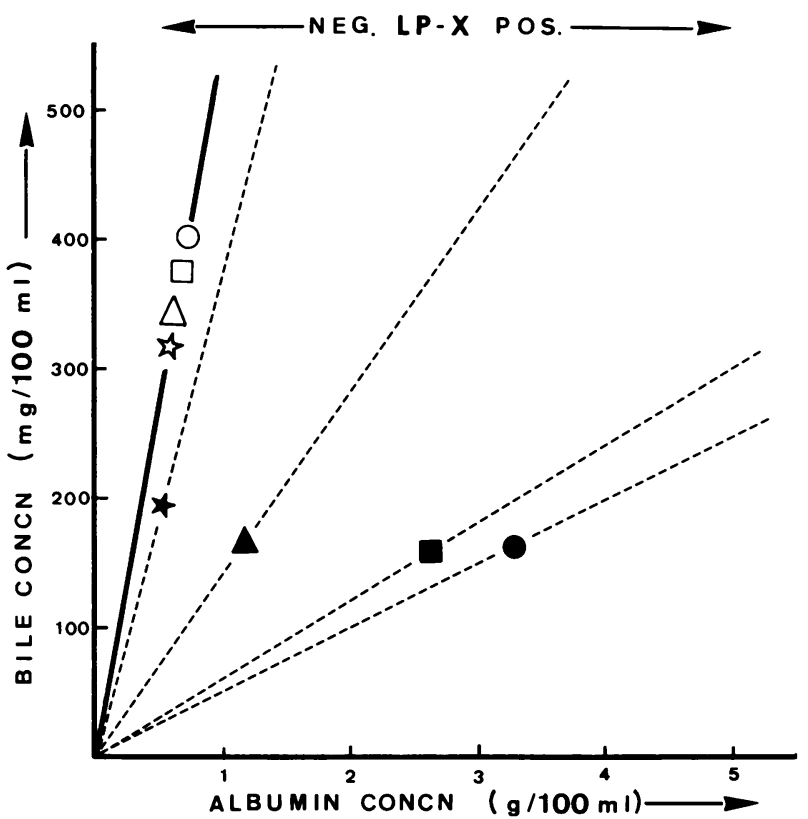

FIGURE 4 Diagram of the required bile/albumin ratio for in vitro LP-X formation. Various native bile samples before (closed symbols) and after (open symbols) dialysis against water. The lines indicate the minimal albumin concentration required for LP-X formation in comparison to the bile concentration. Marked differences between the various bile samples are apparent before dialysis, while after dialysis all samples are on one line and require less albumin than before dialysis. 

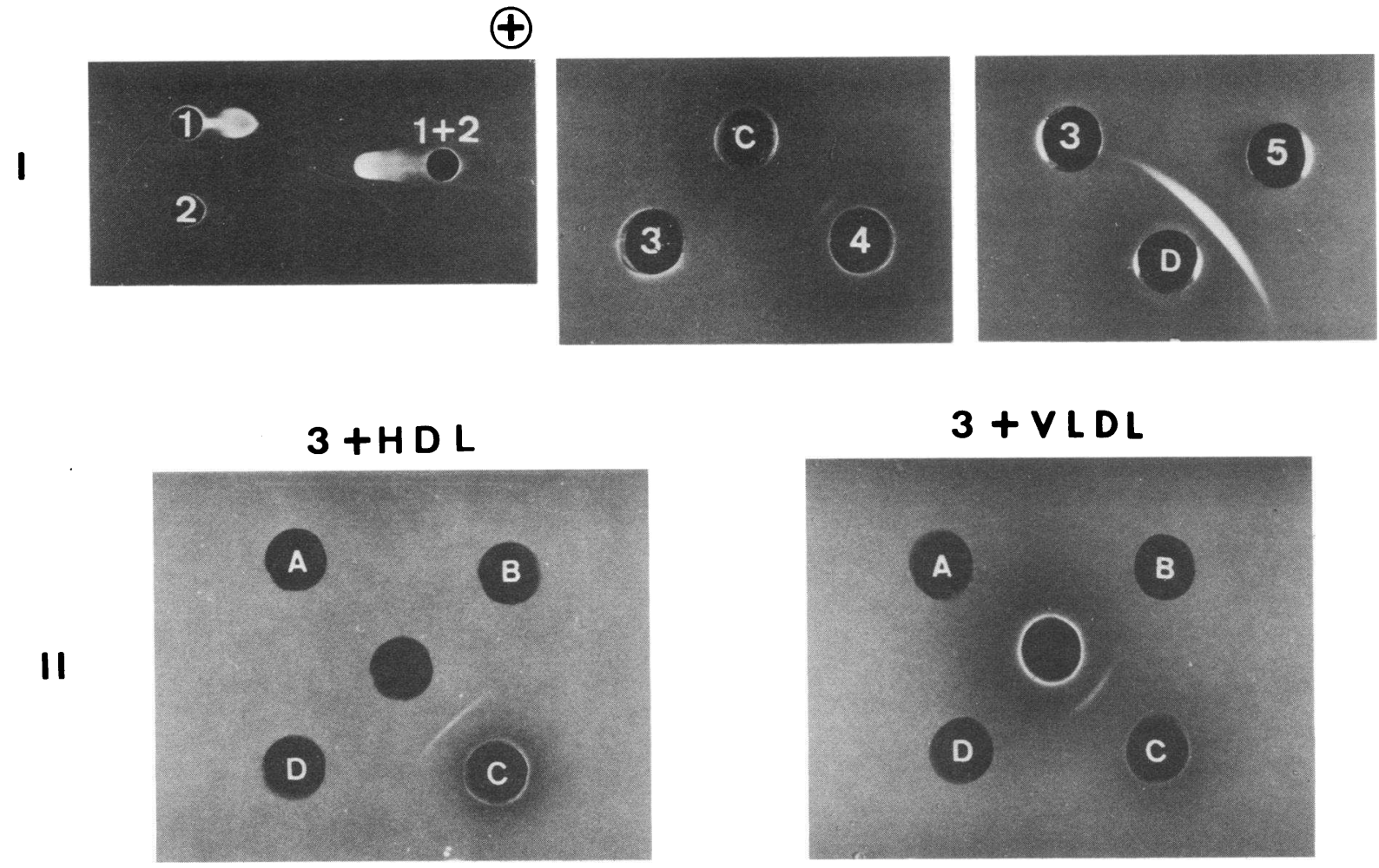

$3+V L D L$

FigURE 5 Electrophoretical and immunochemical characterization of LP-X(a|b). I. Lipoprotein electrophoresis in $1 \%$ agar gel visualized by polyanionic compounds. Native bile (1) shows the typical bile lipoprotein band migrating towards the anode. Albumin shows no lipoprotein band (2). The mixture of 1 and 2 develops the characteristic lipoprotein band for LP-X migrating towards the cathode. No lipoprotein band migrating towards the anode can be visualized in the mixture of bile and albumin. On immunodiffusion the isolated LP-X $(a \mid b)$ (3) shows no reaction against anti-apo $C$ serum $(C)$ as does control-LP-X (4), nor does the isolated LP$\mathrm{X}_{\text {(alb) }}$ (3) react against antialbumin serum (D). However, after partial delipidation of isolated LP-X (alb) (5) a strong immunoreaction against antialbumin serum (D) is apparent. II. After incubation with VLDL or HDL, LP-X $(a 1 b)$ reisolated (in the central well) reveals an immunoreaction with anti-apo $C$ serum (C) but not with anti-apo $A(A)$, anti-apo $B(B)$, or antialbumin serum (D).

of different preparations, the percent incorporation of albumin added varies from bile sample to bile sample depending on its salt concentrations as outlined above (see also Fig. 4).

Incubation of LP-X (alb) with isolated VLDL or HDL (final concentrations 250 and $150 \mathrm{mg} / 100 \mathrm{ml}$, respectively; $2 \mathrm{~h}$, room temperature) leads to a transfer of apo $\mathrm{C}$ from the normal plasma lipoproteins to LP-X (alb), as demonstrated by immunological means (see Fig. 5). A similar transfer to apo A or apo B was not detected with this technique.

As for LP-X (alb) the addition of serum (Fig. 6) to native or water-dialyzed bile revealed a conversion of bile LP into LP-X(ser). However, in contrast to LP-X(alb), LP-X(ser) showed an immunoreaction with antibodies to apo $\mathrm{C}$ in the same manner as described for LP-X isolated from serum (4). The albumin portion of LP-X(ser) can only be demonstrated after partial delipidation or treatment with bile acids as in the case of LP-X (alb) or bile LP (Fig. 6).

The structural integrity of the various forms of LP-X (Fig. 7) seems to depend on the proteins taking part in the formation of the particles as well as on the concentration of bile salts in the fluid. Differences in size and homogeneity of various LP-X preparations can be visualized by electron microscopy and on agar electrophoresis. LP-X (alb) shows somewhat larger and more heterogeneous particles on electron micrographs and migrates further towards the cathode on agar electrophoresis than does LP-X (alb) after incubation with VLDL or LP-X (ser), which seems to be identical to LP-X isolated from serum $(7,8)$.

From the in vitro experiments with the isolated bile LP described here it is evident that LP-X formation 

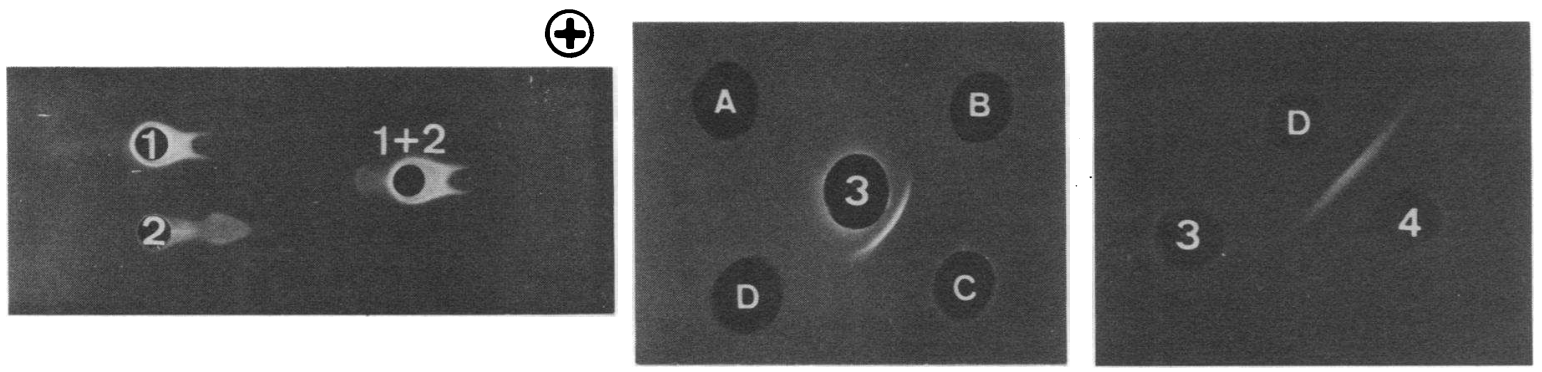

FIGURE 6 Electrophoretic and immunochemical characteristics of LP-X (ser). Addition of LP$\mathrm{X}$-negative serum (1) to native human bile (2) reveals a typical LP-X band migrating towards the cathode on $1 \%$ agar electrophoresis which may be visualized with polyanionic compounds $(0.1 \mathrm{M} \mathrm{MgCl} 2: 0.15 \%$ sodium heparin : $1.3 \% \mathrm{NaCl})(1+2)$. On immunodiffusion the isolated LP-X $($ ser) $(3)$ reacts only against anti-apo $C$ serum $(C)$ and shows no immunoreaction against antisera to apo A (A), apo B (B), or albumin (D). However, after partial delipidation (4) LP-X (ser) reveals a strong immunoreaction with antialbumin serum (D).

takes place when the former is converted into LP-X by the action of albumin in a proper ratio to bile salts. As demonstrated in Fig. 8, isolated bile LP plus albumin alone does not result in LP-X formation and the material develops no electrophoretic mobility (Fig. 8, upper well). However, bile LP resuspended in a solution of bile salts (final concentration $250 \mathrm{mg} / 100 \mathrm{ml}$ ) migrates towards the anode on agar electrophoresis and the addition of albumin in high concentration to this fraction results in immediate complete conversion to LP-X. A certain ratio of albumin to bile salt concentration seems to be required for the observed conversion. This relationship requires further study. A similar relationship also holds for LP-X in whole serum as dem- onstrated in Fig. 9. In vitro addition of bile salts in a concentration of $50-100 \mathrm{mg} / 100 \mathrm{ml}$ (depending on the starting LP-X concentration and possibly also on the plasma albumin concentration) to a LP-X-positive serum results in a disappearance of LP-X when judged on the basis of its typical migration towards the cathode on agar electrophoresis. The particle is still present, however, with a changed electrophoretic mobility towards the anode and a significant increase of bile salts content from $5 \%$ (LP-X normal) to $10 \%$. After adding albumin in increasing concentrations to such a fraction, LP-X with its typical characteristics again appears. This effect cannot be achieved by dialysis of the mixture of LP-X-positive serum plus bile salts, nor is the addi-

TABLE II

Plasma Lipid and LP-X Concentrations in Dog after Insertion of the Bile Duct into the Cava Vein

\begin{tabular}{|c|c|c|c|c|c|}
\hline & Phospholipid & $\begin{array}{c}\text { Total } \\
\text { cholesterol }\end{array}$ & $\frac{\text { Total cholesterol }}{\text { Free cholesterol }}$ & Triglyceride & LP-X \\
\hline & \multicolumn{2}{|c|}{$m g / 100 \mathrm{ml}$} & & \multicolumn{2}{|c|}{$m g / 100 m l$} \\
\hline Before operation & 400 & 150 & 3.58 & 63 & - \\
\hline \multicolumn{6}{|l|}{ After operation } \\
\hline $30 \mathrm{~min}$ & 380 & 125 & 2.60 & 79 & - \\
\hline $1 \mathrm{~h}$ & 359 & 124 & 2.57 & 76 & - \\
\hline $2 \mathrm{~h}$ & 421 & 122 & 2.45 & 73 & - \\
\hline $3 \mathrm{~h}$ & 434 & 132 & 2.55 & 82 & 5 \\
\hline $4 \mathrm{~h}$ & 505 & 148 & 2.43 & 84 & 25 \\
\hline $5 \mathrm{~h}$ & 553 & 165 & 2.46 & 82 & 40 \\
\hline $6 \mathrm{~h}$ & 558 & 170 & 2.44 & 86 & 62 \\
\hline $7 \mathrm{~h}$ & 566 & 193 & 2.61 & 90 & 66 \\
\hline $8 \mathrm{~h}$ & 561 & 196 & 2.69 & 74 & 64 \\
\hline $12 \mathrm{~h}$ & 480 & 171 & 2.28 & 85 & 30 \\
\hline $17 \mathrm{~h}$ & 506 & 174 & 2.24 & 89 & 25 \\
\hline $20 \mathrm{~h}$ & 529 & 174 & 2.22 & 85 & 23 \\
\hline $26 \mathrm{~h}$ & 533 & 168 & 1.91 & 83 & 20 \\
\hline
\end{tabular}




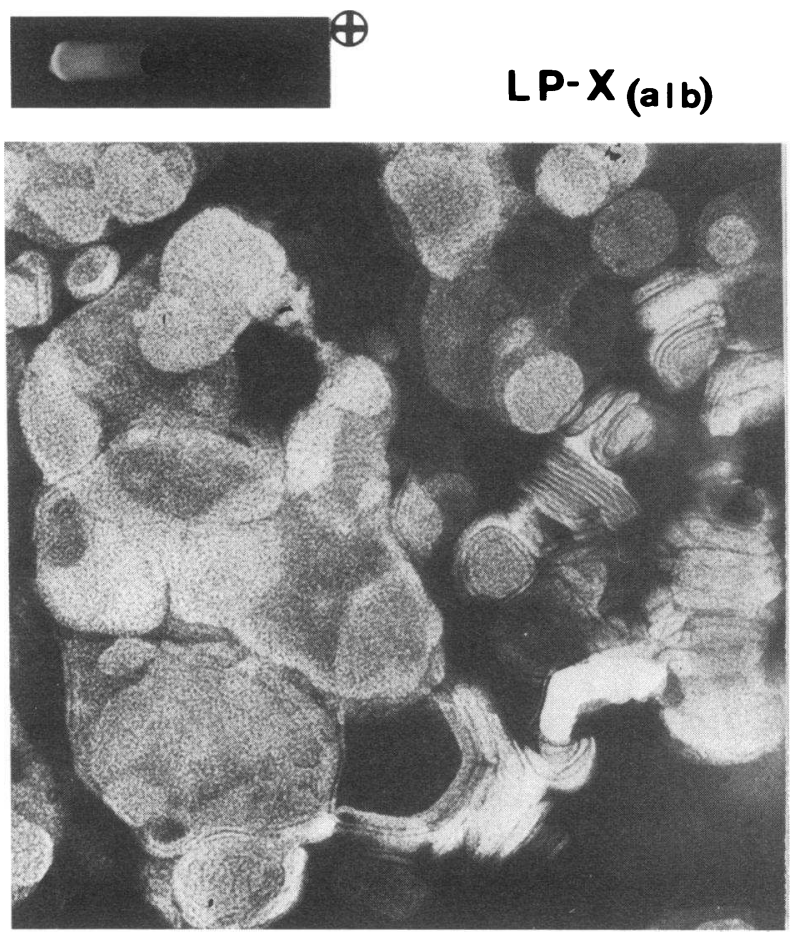

$\overline{0.1 \mu \mathrm{m}}$
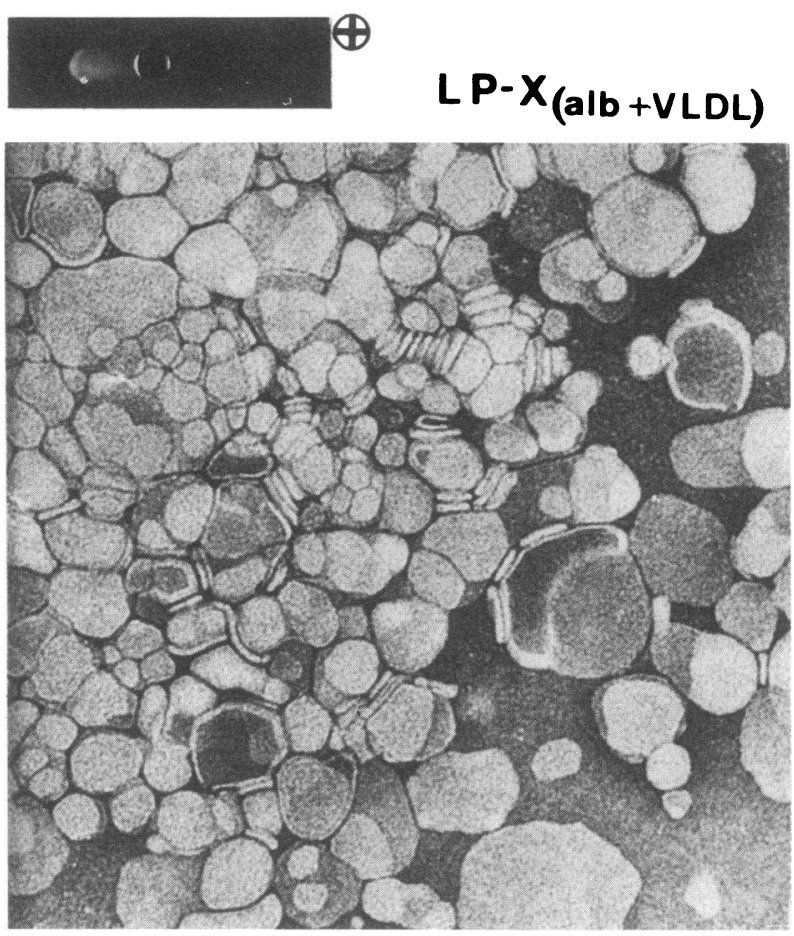

$0.1 \mu \mathrm{m}$

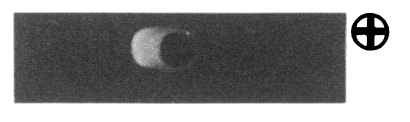

\section{LP-X (ser)}

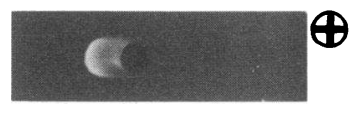

L P - X
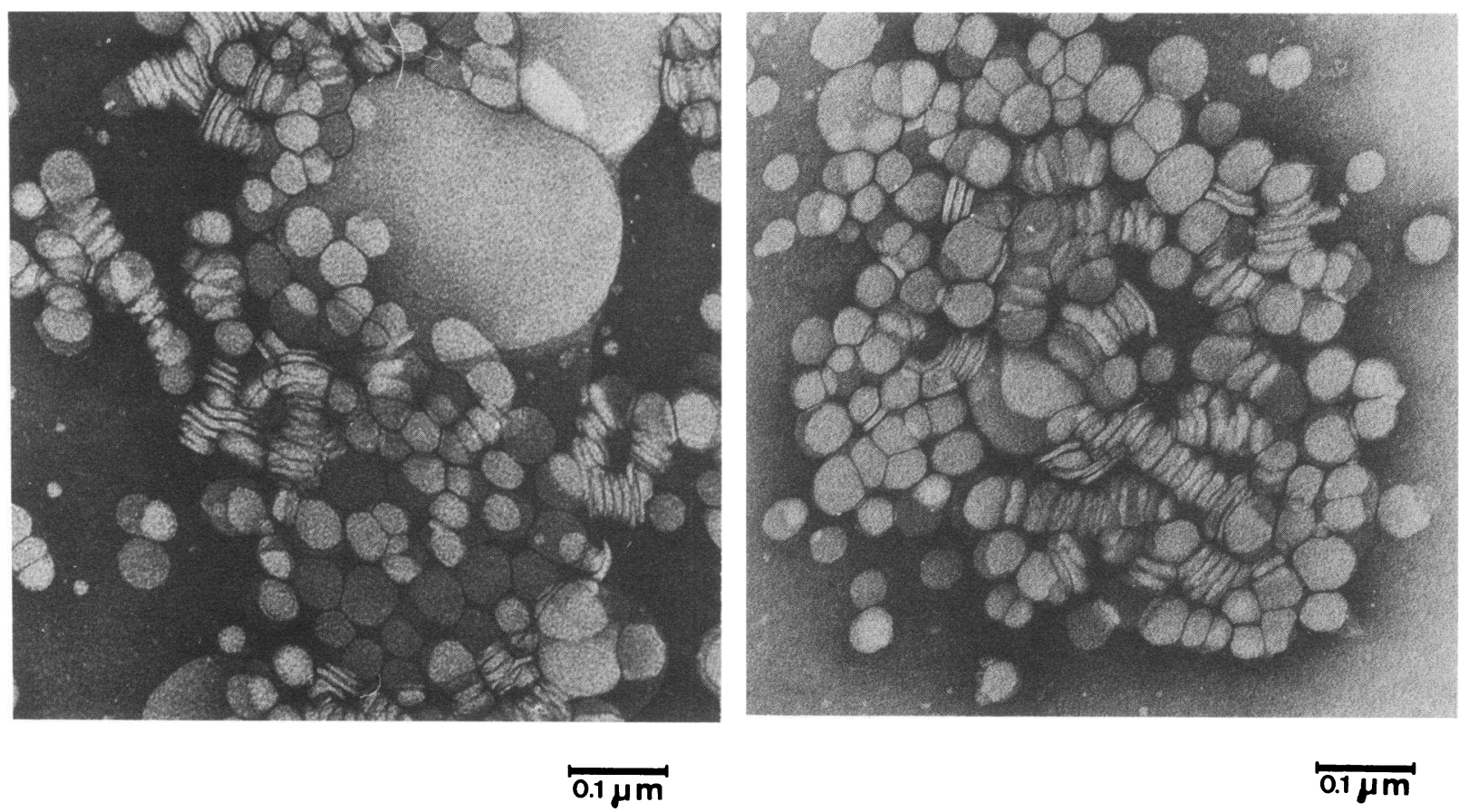

$\overline{0.1 \mu \mathrm{m}}$ 
tion of albumin to a LP-X-negative serum plus bile salts followed by LP-X formation.

From all these in vitro experiments it seemed justified to expect that LP-X is formed when the bile LP, usually excreted from the liver, refluxes into the blood because of cholestatic conditions. This hypothesis is supported by our animal experiments (Fig. 10 and Table II). After insertion of the common bile duct into the vena cava of dogs LP-X was demonstrated as early as $3 \mathrm{~h}$ after operation in four animals treated in this way. A rapid increase in LP-X concentration during the first $8 \mathrm{~h}$ is paralleled by an increase in both cholesterol and phospholipids. Plasma triglycerides remain almost unchanged during the course of the experiment. The initial rapid increase of LP-X and cholesterol is followed by a period of decreasing concentrations. Although there is at present no explanation for this observation it is interesting to note that the increase in phospholipids and cholesterol is considerably greater than one would expect or calculate from the bile lipids entering the blood. The increase in LP-X in cholestasis corresponds to the refluxed bile lipids. The unexpectedly high increase in phospholipids and cholesterol in relation to the refluxed bile lipids under experimental cholestasis was demonstrated earlier $(33,34)$.

\section{DISCUSSION}

We have previously demonstrated that LP-X appears in appreciable amounts in dog and rat plasma within the first $24 \mathrm{~h}$ after surgical ligation of the common bile duct $(35,36)$. The same time seems to be necessary for LP-X to appear in patients with extrahepatic biliary obstruction (11). Our results suggest that LP-X formation is not dependent upon a new metabolic pathway but rather derives from a pathophysiological process closely related to cholestasis.

It has been demonstrated that during extrahepatic biliary obstruction bile passes from the bile duct to the hepatic lymphatics, from there to the thoracic lymphatic duct and finally into the bloodstream (37). The lipid composition of LP-X isolated from serum differs significantly from normal plasma lipoproteins but shows great similarity to the lipids found in normal bile (9). Almost all of the biliary cholesterol exists in unesterified form as in LP-X. Phospholipid concentration is approximately twice as high as the concentration of cholesterol in both bile and LP-X. Even the phosphatide distribution of bile is very similar to that of the phosphatides

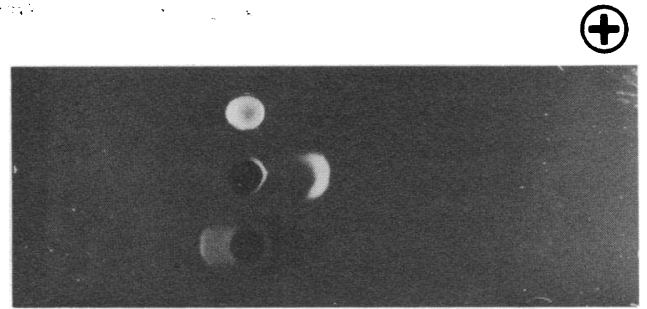

Figure $8 \quad 1 \%$ agar electrophoresis of isolated and treated bile LP. The lipoprotein bands are visualized by polyanion precipitation $\left(0.1 \mathrm{M} \mathrm{MgCl}_{2}: 0.15 \%\right.$ sodium heparin: $1.3 \%$ $\mathrm{NaCl}$ ). Bile LP (upper well) does not develop any electrophoretic migration. Bile LP after addition of bile salts (250 $\mathrm{mg} / 100 \mathrm{ml}$ ) (middle well) migrates towards the anode as does native bile LP (see Fig. 1). Addition of albumin (12 $\mathrm{g} / 100 \mathrm{ml}$ ) to the mixture of bile LP plus bile salts (lower well) leads to LP-X formation.

present in LP-X. Moreover, the phospholipid fatty acids of hepatic bile and those of LP-X show a close similarity (38). These facts taken together may suggest a common origin and relationship of the bile lipids and LP-X.

In this study we have demonstrated that in native bile the lipids are organized in the form of a lipoprotein which can be isolated. This is in agreement with recent reports from different laboratories $(32,39-42)$. This bile LP revealed a lipid composition almost identical to LP-X but differed from LP-X in protein/lipid ratio and immunological and electrophoretic characteristics. Albumin is the major or the only protein portion of bile LP. However, it is detectable only in bile-acid solution or after delipidation. Bile lipoprotein can be converted into LP-X by adding albumin or serum to native bile and the addition of bile salts to a fraction containing LP-X results in formation of bile LP particles. This, plus the fact that only a few hours after experimental connection of the bile duct with the vena cava LP-X appears in the serum of dogs, strongly suggests that the bile LP is a precursor lipoprotein for LP-X. All in vitro experiments as well as the animal model are in agreement with the concept that this conversion is dependent only on cetrain physicochemical requirements and independent of any active metabolic process. It appears that bile acids in low concentrations are necessary to make the bile LP accessible for the incorporation of more albumin, which is important for developing and maintaining the LP-X characteristics. The albumin concentration of normal bile (43) is apparently

Figure 7 Electrophoretic (1\% agar gel) and electron microscopic comparison of various forms of LP-X. LP-X (alb) shows somewhat larger and more heterogeneous particles on electron micrographs and migrates further towards the cathode on agar electrophoresis than does LP$\mathrm{X}_{(\mathbf{a}) \mathbf{b})}$ after incubation with VLDL or LP-X $\mathrm{X}_{(\mathbf{s e r})}$, which seemed to be almost identical with LP-X isolated from serum of cholestatic patients. 


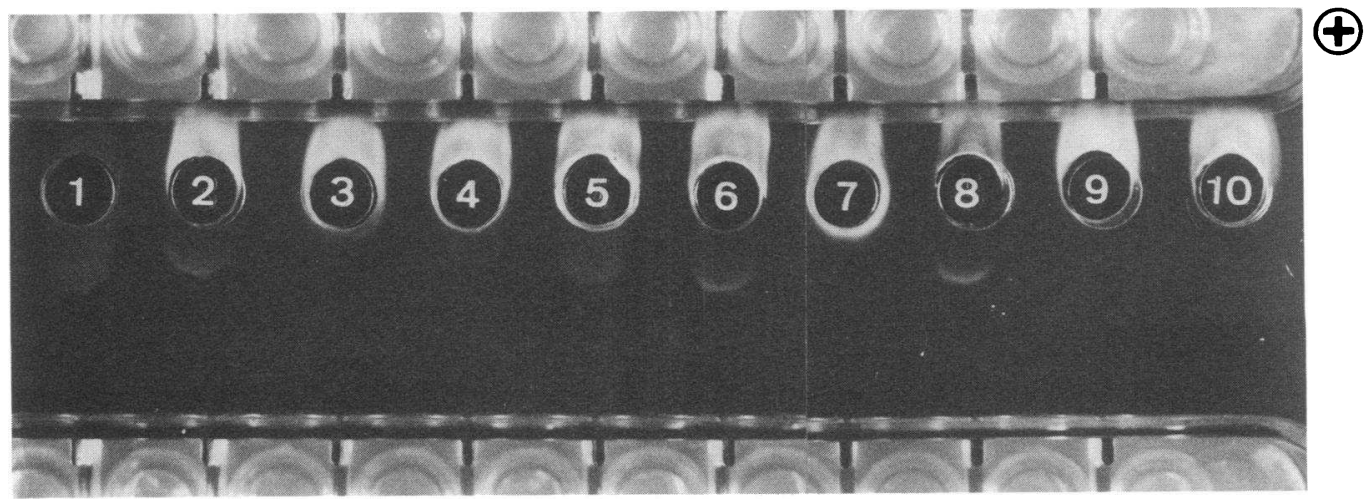

FIGURE 9 Agar electrophoresis (Rapidophor system). (1) Control (LP-X-positive) serum of the test kit; (2) LP-X-positive serum from a patient with extrahepatic biliary obstruction; (3) the same sample as in (2) after addition of bile salts (final concentration $50 \mathrm{mg} / 100 \mathrm{ml}$ ); $(4-6)$ the same samples as in (3) after addition of increasing amounts $(5,8$, and $15 \mathrm{~g} / 100 \mathrm{ml})$ of human albumin; (7) same sample as in (3) after dialysis; (8) the same sample as in (2) after addition of albumin $(5 \mathrm{~g} / 100 \mathrm{ml})$; (9) LP-X-negative serum after addition of albumin $(5 \mathrm{~g} / 100 \mathrm{ml})$; (10) LP-X-negative serum after addition of bile salts $(50 \mathrm{mg} / 100 \mathrm{ml})$ and albumin $(5 \mathrm{~g} / 100 \mathrm{ml})$.

too low in absolute terms and in relation to bile acid concentration to make LP-X formation possible.

High bile salt concentration may convert LP-X back into a bile LP-like particle, even in an environment of
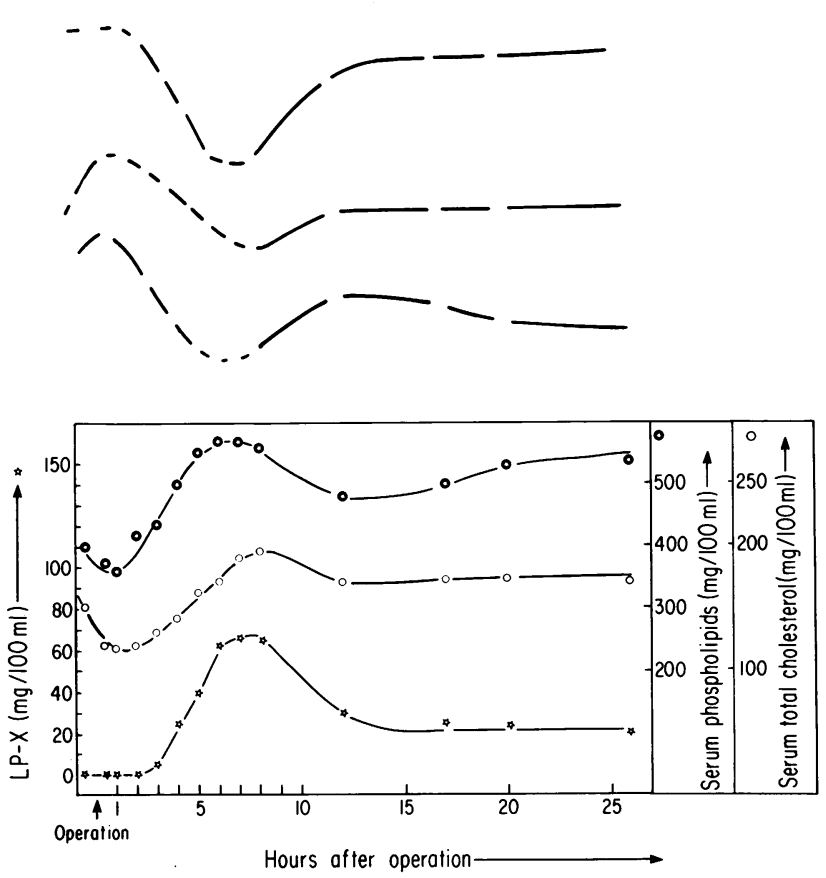

Figure 10 Representative curves of plasma phospholipids, cholesterol, and LP-X concentrations after insertion of the bile duct into the vena cava in a dog. A marked increase in LP-X concentration during the first $8 \mathrm{~h}$ is paralleled by an increase of both cholesterol and phospholipid. Curves very similar to the presented were found in three other animal experiments. high albumin concentration as is found in serum. Therefore, LP-X found in the plasma of subjects with cholestatic liver disease is most likely to be derived from conversion of the refluxing bile LP in contact with the required amounts of albumin. The exact conditions necessary for this conversion will have to be established in additional experiments. It is also an open question whether this conversion takes place only when bile LP enters the circulation or when it comes into contact with plasma albumin at any site. For example, such a conversion may also take place in the liver if the required physicochemical conditions obtain. Evidence along these lines was given by electron microscopic demonstration of LP-X-like particles in the bile ducts of mice after ligation but without cholestasis (44). Our results strongly indicate that albumin is an essential requirement for maintenance of the unique physicochemical characteristics of LP-X. The apo $\mathrm{C}$ protein portion of LP-X as well, as other apolipoproteins which may occasionally be detected in LP-X after isolation from the serum of cholestatic liver disease patients, seems to be of minor importance with respect to the structural integrity of LP-X, although as demonstrated in this study, they may have some stabilizing effect on the particle. This would be in agreement with recombination studies of apolipoproteins with lipids which have consistently indicated higher stability with increased apoprotein concentration $(45,46)$.

\section{ACKNOWLEDGMENTS}

The electron micrographs were kindly performed by $\mathrm{Dr}$. Aumüller, Anatomisches Institut der Universität, Heidelberg.

The study was supported by grants from the Deutsche Forschungsgemeinschaft SFB 90; D-8. 


\section{REFERENCES}

1. Russ, E. M., J. Raymunt, and D. P. Barr. 1956. Lipoproteins in primary biliary cirrhosis. J. Clin. Invest. 35 : 133-144.

2. Switzer, S. 1967. Plasma lipoproteins in liver disease. I. Immunologically distinct low-density lipoproteins in patients with biliary obstruction. J. Clin. Invest. 46: 1855-1866.

3. Seidel, D., P. Alaupovic, and R. H. Furman. 1969. A lipoprotein characterizing obstruction jaundice. I. Method for quantitative separation and identification of lipoproteins in jaundiced subjects. J. Clin. Invest. 48: 1211-1223.

4. Seidel, D., P. Alaupovic, R. H. Furman, and W. J. McConathy. 1970. A lipoprotein characterizing obstructive jaundice. II. Isolation and partial characterization of the protein moieties of low density lipoproteins. $J$. Clin. Invest. 49 : 2396-2407.

5. Picard, J., and D. Veissière. 1970. Separation des lipoprotéines sériques anormales dans la cholestase. $C . R$. Acad. Sci. (Paris) Ser. D. 270: 1845-1848.

6. Mills, G. L., D. Seidel, and P. Alaupovic. 1969. Ultracentrifugal characterization of a lipoprotein occurring in obstructive jaundice. Clin. Chim. Acta. 26: 239-244.

7. Hamilton, R. L., R. J. Havel, J. P. Kane, E. A. Blaurock, and T. Sata. 1971. Cholestasis : lamellar structure of the abnormal human serum lipoprotein. Science (Wash. D. C.). 172: 475-478.

8. Seidel, D., B. Agostini, and P. Müller. 1972. Structure of an abnormal plasma lipoprotein (LP-X) characterizing obstructive jaundice. Biochim. Biophys. Acta. 260: 146-152.

9. Quartfordt, S. H., H. Oelschlaeger, and W. R. Krigbaum. 1972. Liquid crystaline lipid in the plasma of humans with biliary obstruction. J. Clin. Invest. 51: 1979-1988.

10. Wieland, H., and D. Seidel. 1973. Eine neue und vereinfachte Methode zum Nachweis des LP-X, eines cholestasespezifischen Lipoproteins. Dtsch. Med. Wochenschr. 98: 1474-1475.

11. Seidel, D., E. A. Schmitt, and P. Alaupovic. 1970. Ein abnornomes Low-density-Lipoprotein bei Cholestase. II. Beteutung in der Differential diagnose des Ikterus. Dtsch. Ged. Woschenschr. 95 : 1805-1809.

12. Poley, J. R., P. Alaupovic, W. J. McConathy, D. Seidel, C. C. Roy, and A. Weber. 1973. Diagnosis of extrahepatic biliary obstruction in infants by immunochemical detection of LP-X and modified ${ }^{133}$ I-Rose Bengal excretion test. J. Lab. Clin. Med. 81 : 325-341.

13. Seidel, D., H. Gretz, and C. Ruppert. 1973. Significance of the LP-X test in differential diagnosis of jaundice. Clin. Chem. 19: 86-91.

14. Prexl, H-J., and W. Petek. 1973. Die Bedeutung des Lipoprotein-X und der Serumcholesterase in der präoperativen Diagnostik des Verschlussikterus. Chirurg. $44: 310-313$.

15. Ritland, S., J. P. Blomhoff, K. Elgjo, and E. Gjone. 1973. Lipoprotein-X (LP-X) in liver disease. Scand. J. Gastroenterol. 8: 155-160.

16. Vergani, C., M. Pietrogrande, M. C. Grondona, and M. Pizzolato. 1973. Study of the abnormal lipoprotein$\mathrm{X}$ in obstructive and non-obstructive jaundice. Clin. Chim. Acta. 48: 243-248.

17. Rosti, D., and C. G. De Gaspari. 1974. Valorè diagnostico della LP-X negli itteri neonatali. Minerva Pediatr. 26 : 582-585.
18. Mayer, K. 1975. Der Wert der LP-X-Bestimmung zur Erfassung einer Cholestase. In Jahrestagung der Deutschen, Österreichischen und Schweizerischen Gesellschaft für klinische Chemie. Freiburg, W. Germany. März 1613. (Abstr.)

19. Lachmann, D., H. E. Bugaher-Gleitman. G. Eder, und A. Pollak. 1975. LP-X-Untersuchungen bei Neugeborenen und Frühgeburten. In Jahrestagung der Deutschen, Österreichischen und Schweizerischen Gesellschaft für klinische Chemie. Freiburg, W. Germany. März 16-18. (Abstr.)

20. Ober, M., and I. Witt. 1975. Lipoprotein-X (LP-X) bei Neugeborenen: Gehäuftes Auftreten ohne nachweisbare Cholestase. In Jahrestagung der Deutschen, Österreichischen und Schweizerischen Gesellschaft für klinische Chemie, Freiburg, W. Germany. März 16-18. (Abstr.)

21. Fischer, M., C. Falkensammer, G. Baronach, S. Waketuch, O. Kronberger, and H. Schnack. 1975. Zur Diagnose der Cholestase: Lipoprotein-X (LP-X). Wien. Klin. Wochenschr. $87:$ 524-531.

22. Fellin, R., E. Manzato, S. Zotti, P. Balestrieri, and L. Chieco-Bianchi. 1976. Study of correlations between lipoprotein-X and some hepatic enzymes in the diagnosis of cholestasis. Rendiconti di Gastroenterologia (Pozzi Ed., Rome). Abstr. In press.

23. Seidel, D., E. Gjone, J. P. Blomhoff, and H. P. Geisen. 1974. Plasma lipoproteins in patients with familial plasma lecithin : cholesterol acyltransferase (LCAT) deficiency. Studies on the apolipoprotein composition of isolated fractions with identification of LP-X. In Lipid Metabolism, Obesity and Diabetes Mellitus: Impact upon Atherosclerosis. George Thieme Verlag KG., Stuttgart, W. Germany. 6-11.

24. Torsvik, H., K. Berg, H. N. Magnani, W. J. McConathy, P. Alaupovic, and E. Gjone. 1972. Identification of the abnormal cholestatic lipoprotein (LP-X) in familial lecithin: cholesterol acyltransferase deficiency. FEBS (Fed. Eur. Biochem. Soc.) Lett. 24: 165-168.

25. McConathy, W. J., P. Alaupovic, M. D. Curry, H. N. Magnani, H. Torsvik, K. Berg, and E. Gjone. 1973. Identification of lipoprotein families in familial lecithincholesterol acyltransferase deficiency. Biochem. Biophys. Acta. 326: 406-418.

26. Neubeck, W., and D. Seidel. 1975. Direct method for measuring lipoprotein- $\mathrm{X}$ in serum. Clin. Chem. 21: 853856.

27. Noble, R. P. 1968. Electrophoretic separation of plasma lipoproteins in agarose gel. J. Lipid Res. 9: 693-700.

28. Seidel, D., H. Wieland, and C. Ruppert. 1973. Improved techniques for assessment of plasma lipoprotein patterns. I. Precipitation in gels after electrophoresis with polyanionic compounds. Clin. Chem. 19: 737-739.

29. Müller, P., R. Fellin, J. Lambrecht, B. Agostini, H. Wieland, W. Rost, and D. Seidel. 1974. Hypertriglyceridaemia secondary to liver disease. Eur. J. Clin. Invest. $4: 419-428$.

30. Russel, I. S., and W. Burnett. 1963. The proteins of human bile. Gastroenterology. 45 : 730-739.

31. Howell, J. I., J. A. Lucy, R. C. Pirola, and I. A. D. Bouchier. 1970. Macromolecular assemblies of lipid in bile. Biochim. Biophys. Acta. 210: 1-6.

32. Nalbone, G., H. Lafont, N. Domingo, D. Lairon, G. Pautrat, and J. Hauton. 1973. Ultramicroscopic study of the bile lipoprotein complex. Biochemie (Paris). 55: 1503-1506.

33. Byers, S. O., and M. Friedman. 1952. Observations concerning the production and excretion of cholesterol in 
mammals. V. The relation of biliary retention of cholesterol, distension of the biliary tract, the shunting of bile to the vena cava, and the removal of the gastrointestinal tract to the hypercholesterolemia consequent on biliary obstruction. J. Exp. Med. 95: 19-24.

34. Weis, H. J., and E. U. Baas. 1972. Causes of hypercholesterolemia after biliary ligation in the rat. In Bile Acids in Human Disease. P. Back and W. Gerok, editors. Friedrich-Karl Schattaur-Verlag, Stuttgart, W. Germany. 121-123.

35. Müller, P., U. Fauser, R. Fellin, H. Wieland, and D. Seidel. 1973. Isolation and characterization of lipoprotein-X (LP-X) from canine. FEBS (Fed. Eur. Biochem. Soc.) Lett. 38: 53-56.

36. Seidel, D., H. U. Büff, U. Fauser, and U. Bleyl. 1976. On the metabolism of lipoprotein-X (LP-X). Clin. Chim. Acta. 66: 135-207.

37. Popper, H. 1968. Cholestasis. Annu. Rev. Med. 19: 39-55.

38. Picard, J., F. Veissiere, F. Voyer, and G. Bereziat. 1972. Composition en acides gras des phospholipides dans les lipoproteines sériques anormales de la cholestase. Clin. Chim. Acta. 36: 247-250.

39. Lairon, D., H. Lafont, and J-C. Hauton. 1972. Lack of mixed micelles bile salt-lecithin-cholesterol in bile and presence of a lipoprotein complex. Biochimie (Paris). 54: 529-530.

40. Lafont, H., D. Lairon, N. Domingo, G. Nalbone, and
J. C. Hauton. 1974. Does a lecithin-polypeptide association in bile originate from membrane structural subunits? Biochimie (Paris). 56: 465-468.

41. Gregory, D. H., R. Z. Vlahcevic, P. Schatzki, and L. Swell. 1975. Mechanism of secretion of biliary lipids. I. Role of bile canalicular and microsomal membranes in the synthesis and transport of biliary lecithin and cholesterol. J. Clin. Invest. 55: 105-114.

42. Quarfordt, S. H., H. Oelschlaeger, W. R. Krigbaum, L. Jakoi, and R. Davis. 1973. Effect of biliary obstruction on canine plasma and biliary lipids. Lipids. 8: 522530.

43. Dive, C., and J. F. Heremans. 1974. Nature and origin of the proteins of bile. I. A comparative analysis of serum and bile proteins in man. Eur. J. Clin. Invest. 4 : 235-239.

44. Stein, O., M. Alkan, and Y. Stein. 1973. Obstructive jaundice lipoprotein particles studied in ultrathin sections of livers of bile duct-ligated mice. Lab. Invest. 29: 166-172.

45. Hoff, H. F., J. D. Morrisett, and A. M. Gotto, Jr. 1973. Interaction of phosphatidylcholine and apolipoproteinalanine: electron microscopic studies. Biochim. Biophys. Acta. 296: 653-660.

46. Forte, T., E. Gong, and A. V. Nichols. 1974. Interaction by sonication of C-apolipoproteins with lipid: an electron microscopic study. Biochim. Biophys. Acta. 337: 169-183. 